\title{
Safety and Technical Efficacy of Tumescent Anesthesia in Radiofrequency Ablation for Thyroid Nodules Close to the Surrounding Structure
}

\author{
Tae Ik Eom, Byung Seup Kim \\ Department of Surgery, HiU Clinic, Suwon, Korea
}

Received December 27, 2018

Revised January 25, 2019

Accepted February 11, 2019
Correspondence to:

Byung Seup Kim

Department of Surgery, HiU Clinic,

170 Gwongwang-ro, Paldal-gu,

Suwon 16488, Korea

Tel: + 82-31-221-1205

Fax: + 82-31-225-1207

E-mail: kgiseup@naver.com
Purpose: This study estimated the changes in distance between a thyroid nodule and the surrounding structures after tumescent anesthesia in radiofrequency ablation (RFA) for a benign thyroid nodule.

Methods: Between January 2015 and December 2017, the sonogram images in patients treated with thyroid RFA were reviewed retrospectively. Patients without images immediately after tumescent anesthesia or treated with RFA for carcinoma were excluded. The $0.2 \%$ saline mixed lidocaine as a tumescent solution was injected into the anterior, posterior, and lateral capsules of the thyroid and thyroid-trachea junction. In a sonogram image, the distances between the posterior margin of the nodule and above the longus colli muscle, between the medial margin of the nodule and trachea, and between the lateral margin of the nodule and common carotid artery (CCA) were measured before and after tumescent anesthesia.

Results: Tumescent anesthesia was technically successful in all 133 patients (100\%) and the analgesic effect was also obtained successfully. No complications related to tumescent anesthesia were observed. The average distance between the posterior margin of the nodule and the longus colli muscle increased by $4.2 \pm 2.4 \mathrm{~mm}$. The average distance between the medial margin of the nodule and trachea increased by $2.2 \pm 1.9 \mathrm{~mm}$. The average distance between the lateral margin of the nodule and CCA increased by $4.6 \pm 3.0 \mathrm{~mm}$.

Conclusion: Tumescent anesthesia is a safe and useful method for secure a safe distance (over $2 \mathrm{~mm}$ ) in RFA for thyroid nodules close to the surrounding structure.

Keywords: Radiofrequency ablation, Thyroid, Anesthesia, Injection

\section{INTRODUCTION}

Radiofrequency ablation (RFA) has been widely used to treat benign thyroid nodules. (1-3) The large thyroid nodule is located closely to critical structures such as the esophagus, trachea, recurrent laryngeal nerve (RLN), common carotid artery (CCA), and the vagus nerve. Several studies have reported the com- plications related to thyroid RFA such as nerve or trachea injury.(4-6) In RFA, the ablated lesion spreads out to an oval shape around the tip. (7) If the RFA needle tip is not located on the periphery of the nodule for the fear of an injury of the surrounding structure, the nodule can re-grow from the viable cell. (8)

Tumescent injection has been known as a useful 
method in separating the tumor from the adjacent critical structures in RFA for the hepatic tumor. (9) A tumescent solution was injected to a narrow space between the structures, thereby increasing the distances between the structures. With the assistance of a tumescent solution, the safe distance for RFA was sufficiently secured. In tumescent anesthesia, the tumescent effect is combined with the local analgesic effect using a diluted solution of local analgesics. (10) However, previous studies did not estimate the extent to which the distance between nodule and adjacent structure can increase by tumescent anesthesia. In the present study, we estimated the changes of distance between structures after tumescent anesthesia and evaluated the safety and technical efficacy in RFA for a benign thyroid nodule.

\section{METHODS}

\section{Patient and sonogram image review}

The Public Institutional Review Board Designated by Ministry of Health and Welfare approval to conduct the present study (P01-201812-21-004). Between January 2015 and December 2017, a total of 171 patients with thyroid nodules were consecutively treated with ultrasound guided percutaneous RFA at our institution. The indication of RFA for thyroid nodule included a growing, palpable or symptomatic benign thyroid nodule with discomfort or a cosmetic problem. Although the benign thyroid nodule was not too large in size, the RFA was performed in the cases of the patients anxiety. Prior to RFA, all thyroid nodules were confirmed by fine needle aspiration cytology or core needle biopsy. The RFA for micro-papillary thyroid carcinoma was performed, after consent was obtained, in the elder patients with a high operation risk. The patients treated with RFA for thyroid cancer and metastatic lymph node were excluded from the sample. The sonogram images were retrospectively reviewed using the Picture Archiving and Communication System (PACS). The patients without sonogram images before and after tumescent anesthesia were also excluded.

In a sonogram image, the distances between posterior margin of the nodule and above the longus colli muscle, between medial margin of the nodule and trachea, and between lateral margin of the nodule and CCA were measured before and after tumescent anesthesia using the PACS tool (Fig. 1). The distances before and after tumescent anesthesia were then compared.

The nodule contents were divided into the cystic mass group and the solid mass group. Pure cyst and mainly cystic mass with below 50\% solid portion were classified into the cystic mass group. The solid mass, spongiform mass, and complex echoic mass with above $50 \%$ solid portion were classified into the solid mass group.

The nodule lengths were measured for transverse diameter (TD) and vertical diameter (VD) in the transverse section image, and for longitudinal length

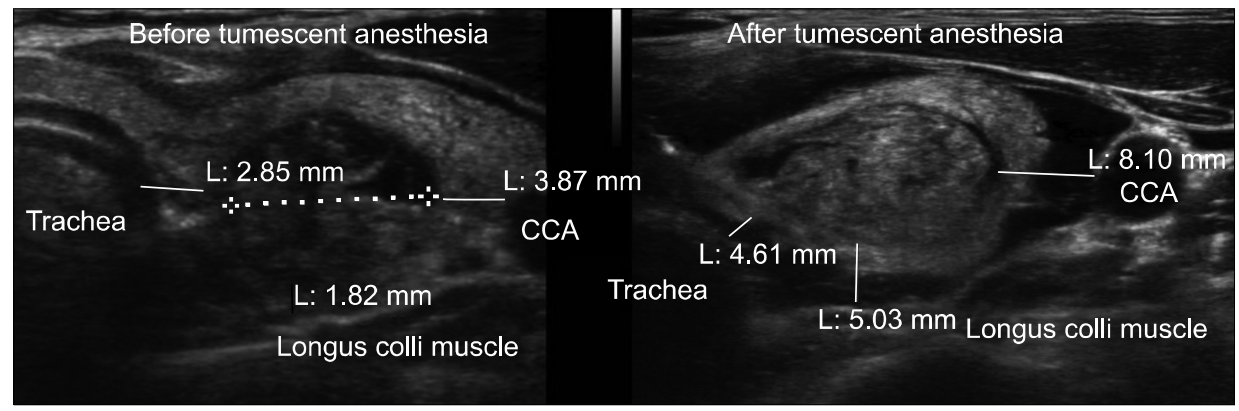

Fig. 1. The distances between nodule and major structures were measured on PACS image before and after tumescent anesthesia using same method. $\mathrm{CCA}=$ common carotid artery. 
(LD) in the longitudinal section image. The volume of nodule was approximated using the following formula: Volume $=\pi \times(\mathrm{TD} \times \mathrm{VD} \times \mathrm{LD}) / 6$.

The RFA time was measured by checking the stored record of time on the sonogram image. The RFA time was considered as only ablation time after completing tumescent anesthesia. We checked the stored record of time immediately after the tumescent injection and immediately after RFA. The time differences were considered as the RFA time.

The records for pain and complications related RFA were checked based on the chart review.

\section{Tumescent anesthesia}

The first procedure of thyroid RFA was the injection of local anesthesia to the skin at the puncture site with $2 \%$ lidocaine. In the next step, $0.2 \%$ saline mixed lidocaine was prepared as the tumescent solution (2\% lidocaine and normal saline, at the ratio 1:9). After skin anesthesia, a 24-gauge $60 \mathrm{~mm}$ long needle was advanced into the interface between the strap muscle and the thyroid anterior capsule under ultrasound guidance using the trans-isthmic approach. Then, over $20 \mathrm{ml}$ of tumescent solution were injected until the anechoic tumescent layer between the strap muscle and the thyroid anterior capsule was seen on the real-time sonogram. Next, the needle was advanced into the interface between the thyroid posterior capsule and the longus colli muscle. The about $40 \mathrm{ml}$ of tumescent solution were injected until the thyroid was shifted upward and medially. Thirdly, the needle was advanced into the interface between CCA and the thyroid lateral capsule. This space already widened to some degree because of the second injected tumescent solution to the posterior capsule. In order to reinforce the safe distance from CCA, an additional tumescent solution was injected between CCA and the lateral thyroid capsule until a more tumescent layer was obtained. Fourthly, the needle was advanced into the interface between thy- roid and trachea and the tumescent solution were slowly injected. Finally, the needle was again advanced into the interface between posterior capsule and the longus colli muscle and the tumescent solution was injected to reinforce the safe distance from RLN. The tumescent solution was injected sufficiently until the tumescent layer was formed, and the thyroid shifted upwards.

\section{RFA procedure}

The procedure was performed using a radiofrequency generator (VIVA RF generator, STARmed, Goyang, Korea) and a peristaltic pump (VIVA pump, STARmed, Goyang, Korea) to lower the tip temperature. We used an 18-gause, monopolar, modified, internally cooled electrode (VIVA, STARmed, Goyang, Korea) with a $1 \mathrm{~cm}$ active tip and a $7 \mathrm{~cm}$ shaft length. The RFA started from the posterior portion of the nodule, because the air bubble near needle masked the tip location under ultrasound. The RFA was terminated when the tumor changed to a transient hyperechoic zone. Conscious sedation was not applied, because the patients' state had to be monitored though verbal questions whether they experienced pain.

\section{Statistical analysis}

The independent t-test was used for continuous variables in order to identify statistically significant associations before and after tumescent anesthesia. A P-value of $<0.05$ was considered to be statistically significant. The analyses were performed using the SPSS version 12.0 (SPSS Inc., Chicago, IL, USA).

\section{RESULTS}

\section{Enrolled patients}

Among 171 patients treated with RFA for the thyroid nodule, 11 patients with micro-papillary thyroid carcinoma and 2 patients with lymph node metastasis 
were excluded in this study. The 25 patients without image immediately after the tumescent injection were also excluded, as we were unable to identify the distance change on the PACS images. Therefore, we reviewed the sonogram images of a total of 133 patients.

\section{Technical feasibility and safety of tumescent anesthesia}

The tumescent injection of $0.2 \%$ diluted lidocaine with normal saline was technically successful in all patients (100\%). All thyroids were shifted after the tumescent injection and the analgesic effect was also successfully obtained. There was no pain during the RFA procedure. However, 12 patients (9.0\%) complained about neck pain after RFA, escaping from anesthesia. They took oral analgesics and improved in 2-3 day. No complications related to tumescent anesthesia were observed. No complications such as skin burn or permanent voice change related to RFA were observed after RFA and 1 year later.

The average distance between posterior margin of the nodule and the longus colli muscle increased by $4.2 \pm 2.4 \mathrm{~mm}$ (from $2.1 \pm 2.1 \mathrm{~mm}$ before to $6.3 \pm 2.7$ $\mathrm{mm}$ after the tumescent injection) $(\mathrm{P}<0.001)$. The average distance between medial margin of the nodule and trachea increased by $2.2 \pm 1.9 \mathrm{~mm}$ (from 1.9 $\pm 2.1 \mathrm{~mm}$ to $4.2 \pm 2.5 \mathrm{~mm})(\mathrm{P}<0.001)$. The average distance between lateral margin of the nodule and CCA increased by $4.6 \pm 3.0 \mathrm{~mm}$ (from $2.1 \pm 2.4 \mathrm{~mm}$ to $6.7 \pm 3.5 \mathrm{~mm})(\mathrm{P}<0.001)$ (Table 1$)$. In the nodules with less than $2 \mathrm{~mm}$ distance from the longus colli muscle, trachea or CCA, the posterior distance increased by $4.8 \pm 2.4 \mathrm{~mm}$ from the longus colli muscle $(\mathrm{n}=90)$, the medial distance increased by $2.4 \pm 1.7$ $\mathrm{mm}$ from trachea $(\mathrm{n}=97)$, and the lateral distance

Table 1. The Distance between Nodule and Structures before and after Tumescent Anesthesia

\begin{tabular}{lcccc}
\hline & Before & After & P-value & Increased distance \\
\hline Nodule - Longus colli m. (post.) & $2.1 \pm 2.1 \mathrm{~mm}$ & $6.3 \pm 2.7 \mathrm{~mm}$ & $<0.001$ & $4.2 \pm 2.4 \mathrm{~mm}$ \\
Nodule - Trachea (med.) & $1.9 \pm 2.1 \mathrm{~mm}$ & $4.2 \pm 2.5 \mathrm{~mm}$ & $<0.001$ & $2.2 \pm 1.9 \mathrm{~mm}$ \\
Nodule - CCA (lat.) & $2.1 \pm 2.4 \mathrm{~mm}$ & $6.7 \pm 3.5 \mathrm{~mm}$ & $<0.001$ & $4.6 \pm 3.0 \mathrm{~mm}$ \\
\hline
\end{tabular}

$\mathrm{m}=$ muscle; $\mathrm{CCA}=$ common carotid artery; post. = posterior; med. = medial; lat. = lateral.

Table 2. The Distance between Nodule and Structures in the Nodules with less than $2 \mathrm{~mm}$ Distance from the Major Structures

\begin{tabular}{lccccc}
\hline & $\mathrm{n}$ & Before & After & P-value & Increased distance \\
\hline Nodule - Longus colli m. (post.) & 90 & $0.9 \pm 0.5 \mathrm{~mm}$ & $5.7 \pm 2.3 \mathrm{~mm}$ & $<0.001$ & $4.8 \pm 2.4 \mathrm{~mm}$ \\
Nodule - Trachea (med.) & 97 & $0.9 \pm 0.6 \mathrm{~mm}$ & $3.3 \pm 1.8 \mathrm{~mm}$ & $<0.001$ & $2.4 \pm 1.7 \mathrm{~mm}$ \\
Nodule - CCA (lat.) & 94 & $0.8 \pm 0.5 \mathrm{~mm}$ & $5.6 \pm 2.9 \mathrm{~mm}$ & $<0.001$ & $4.8 \pm 2.9 \mathrm{~mm}$ \\
\hline
\end{tabular}

$\mathrm{m}=$ muscle; $\mathrm{CCA}=$ common carotid artery; post. = posterior; med. = medial; lat. = lateral.

Table 3. The Change of Distance between Nodule and Structures in Cystic and Solid Nodule after Tumescent Anesthesia

\begin{tabular}{llr}
\hline & \multicolumn{2}{c}{ Increased distance after tumescent anesthesia } \\
\cline { 2 - 3 } & Cyst (n=37) & Solid (n = 96) \\
\hline Nodule - Longus colli m. (post.) & $4.0 \pm 2.4 \mathrm{~mm}$ & $4.3 \pm 2.5 \mathrm{~mm}$ \\
Nodule - Trachea (med.) & $1.8 \pm 1.7 \mathrm{~mm}$ & $2.4 \pm 1.9 \mathrm{~mm}$ \\
Nodule - CCA (lat.) & $4.5 \pm 3.0 \mathrm{~mm}$ & 0.11 \\
\hline
\end{tabular}

$\mathrm{m}=$ muscle; $\mathrm{CCA}=$ common carotid artery; post. = posterior; med. = medial; lat. = lateral. 
Table 4. The Change of Distance between Nodule and Structures in Small and Large Volume of Nodule after Tumescent Anesthesia

\begin{tabular}{lccc}
\hline & \multicolumn{2}{c}{ Increased distance after tumescent anesthesia } & P-value \\
\cline { 2 - 3 } & Volume $<300 \mathrm{~mm}^{3}(\mathrm{n}=23)$ & Volume $\geq 300 \mathrm{~mm}^{3}(\mathrm{n}=110)$ & 0.36 \\
\hline Nodule - Longus colli m. (post.) & $3.8 \pm 2.8 \mathrm{~mm}$ & $4.3 \pm 2.3 \mathrm{~mm}$ & 0.91 \\
Nodule - Trachea (med.) & $2.2 \pm 2.1 \mathrm{~mm}$ & $2.6 \pm 1.8 \mathrm{~mm}$ & 0.15 \\
Nodule - CCA (lat.) & $3.8 \pm 2.4 \mathrm{~mm}$ & $4.8 \pm 3.1 \mathrm{~mm}$ & \\
\hline
\end{tabular}

$\mathrm{m}=$ muscle; $\mathrm{CCA}=$ common carotid artery; post. = posterior; med. = medial; lat. = lateral.

increased by $4.8 \pm 2.9 \mathrm{~mm}$ from CCA $(\mathrm{n}=94)$, respectively (Table 2). The nodule content (cyst vs. solid) and nodule volume (<300 vs. $\geq 300 \mathrm{~mm}^{3}$ ) were not statistically different in terms of the distance from the adjacent structures (Table 3, 4). The RFA time after tumescent anesthesia was $6.2 \pm 3.5$ minute (from 2 to 18 minutes).

The volume reduction rate 1 year post-RFA ranged from $20.1 \%$ to $100 \%$ (mean $\pm \mathrm{SD}, 86.5 \pm 17.1 \%$ ) except for the follow-up loss of 38 patients. We considered less than $5 \mathrm{~mm}$ hypoechoic lesion looking like a scar tissue after RFA 1 year as complete remission. The complete remission rate was $29.5 \%$ except for the follow-up loss of 38 patients.

\section{DISCUSSION}

While the RFA treatment for the benign thyroid nodule was effective, inadvertent complications, such as a thermal injury of the structures adjacent to the nodule, could have occurred. In the situation when the RFA needle tip was not seen under sonogram, the flowing of radiofrequency was very dangerous. Although the needle tip was carefully located in the inside of nodule, an inadvertent thermal injury over the nodule could have occurred due to the extension of ablation to the surrounding area of the needle. Park et al. (11) mentioned that ablation was safe when the tip of the electrode was well positioned within a 1-2 $\mathrm{mm}$ distance from the target nodule margin. Likewise, Cosman et al. (7) mentioned that the needle tip gauge, tip length, generator temperature, and time substantially can affect RFA size and the RFA lesion exceeds the tip length by $1-5 \mathrm{~mm}$. In our experience, the safe distances of over $2 \mathrm{~mm}$ were obtained by tumescent anesthesia in the cases where the tumor was located to the surrounding structure closer than at $2 \mathrm{~mm}$.

The normal saline and local analgesics were used to get the tumescent anesthesia. Lim et al. (12) injected the solution of $5 \%$ dextrose to get the effect of hydrodissection in the treatment for locoregional recurrence from papillary thyroid cancer. Laeseke et al. (13) reported that the normal saline or $5 \%$ dextrose injection had a statistically significant protective effect as compared with the non-pretreated group in terms of protection against unintended thermal injuries from RFA in the treatment of hepatic tumor. When the 5\% dextrose was compared with normal saline, the $5 \%$ dextrose was found to be more effective than saline. The authors mentioned that the normal saline was an ionic fluid, so it could cause frictional heating damage to the adjacent structure due to conduction of electricity. However, the 5\% dextrose does not conduct electricity, so can provide a thermal barrier. Although the protective effect of the saline injection was weaker than that of $5 \%$ dextrose, the saline injection still demonstrated the protective effect. Raman et al.(14) reported that intraperitoneal injection of saline before radiofrequency ablation decreased the frequency and severity of associated diaphragm injury in an animal model. In the present study, we used the normal saline with local analgesics as the tumescent solution, 
as local analgesics were stable in an ionic fluid. To get both the anesthesia and the tumescent effects, using normal saline was required. The issue of the weaker barrier effect of normal saline as compared to that of the $5 \%$ dextrose was overcome, as a larger tumescent solution was injected to obtain the sufficient tumescent layer.

Lim et al.(12) insisted that the tumescent fluid should be injected continuously with the injection needle remaining in the place, as the injected fluid may rapidly spread along the longitudinally arranged neck muscle plane. In the present study, we did not inject tumescent continuously. Immediately after tumescent anesthesia, the injection needle was withdrawn, the ablation needle was rapidly inserted to posterior margin of the nodule, and ablation of posterior margin was started. In our experience, the safe distance was maintained during the ablation for peripheral margin. When the safe distance decreased, an additional tumescent was intermittently injected. Liu et al.(15) injected the 5\% dextrose or saline solution in RFA for hepatic malignancy not continuously, but every 2 minutes. In the present study, we reasoned that an intermittent injection would be tolerable, as the time of ablation for peripheral margin was not long.

The RLN was not identified on sonogram. In the surgical topographic anatomy, the course of RLN is different in the left and the right sides. The location of the left RLN was assumed based on the trachea-esophageal groove, since the left RLN is located in the trachea-esophageal groove. (16) The location of the right RLN was assumed to be not parallel, but obliquely below the thyroid, forming an angle to the trachea. (17) The distance between the nodule and RLN could not be checked, since RLN was not identified in sonogram. The aim of the present study was to identify the increased safe distance. There are thin layers of enclosed thyroid between the thyroid and the recurrent laryngeal nerve. Capsular dis- section along the layers is a useful method to preserve RLN in surgery. (18) We assumed that the tumescent solution was injected into these layers and it would ensure a safe dissection, as in capsular dissection in surgery. We also assumed that the distance between RLN and posterior thyroid margin would followed the same pattern as the increased distance between posterior thyroid margin and the longus colli muscle due to spreading of tumescent solution within the capsular layer.

The present study has several limitations related to its retrospective design. First, we were unable to determine volume of the injected tumescent solution, because the injected volume was not recorded anywhere. In our experience, most RFA were finished with less than $200 \mathrm{ml}$ diluted tumescent of $0.2 \%$ lidocaine $(2 \mathrm{mg} / \mathrm{ml})$. The maximum safe dosages of tumescent lidocaine are $28 \mathrm{mg} / \mathrm{kg}$, so $0.2 \%$ diluted lidocaine less than $200 \mathrm{ml}$ was safe.(19) Second, we could not evaluate the true benefit of tumescent anesthesia with regard to thermal damage, as we could not confirm tissue change by RFA in vivo and could not perform a randomized controlled study. Third, we did not consider the decreasing effect for tumor volume, as our primary goals was to evaluate safety and technical efficacy of tumescent anesthesia.

\section{CONCLUSION}

Our results suggest that tumescent anesthesia is a safe and useful method to secure a safe distance (over $2 \mathrm{~mm}$ ) in RFA for the thyroid nodule very close to the surrounding structure regardless of tumor content and volume.

\section{REFERENCES}

1. Spiezia S, Garberoglio R, Milone F, Ramundo V, Caiazzo C, Assanti AP, et al. Thyroid nodules and related symptoms are stably controlled two years after radiofrequency thermal ablation. Thyroid 2009;19: 
$219-25$.

2. Cesareo R, Pasqualini V, Simeoni C, Sacchi M, Saralli E, Campagna G, et al. Prospective study of effectiveness of ultrasound-guided radiofrequency ablation versus control group in patients affected by benign thyroid nodules. J Clin Endocrinol Metab 2015;100: $460-6$.

3. Jeong WK, Baek JH, Rhim H, Kim YS, Kwak MS, Jeong HJ, et al. Radiofrequency ablation of benign thyroid nodules: safety and imaging follow-up in 236 patients. Eur Radiol 2008;18:1244-50.

4. Ha EJ, Baek JH, Lee JH, Kim JK, Shong YK. Clinical significance of vagus nerve variation in radiofrequency ablation of thyroid nodules. Eur Radiol 2011;21:2151-7.

5. Baek JH, Lee JH, Sung JY, Bae JI, Kim KT, Sim J, et al.; Korean Society of Thyroid Radiology. Complications encountered in the treatment of benign thyroid nodules with US-guided radiofrequency ablation: a multicenter study. Radiology 2012;262:335-42.

6. Wang JF, Wu T, Hu KP, Xu W, Zheng BW, Tong G, et al. Complications following radiofrequency ablation of benign thyroid nodules: a systematic review. Chin Med J (Engl) 2017;130:1361-70.

7. Cosman ER Jr, Dolensky JR, Hoffman RA. Factors that affect radiofrequency heat lesion size. Pain Med 2014;15:2020-36.

8. Kim JH, Baek JH, Lim HK, Ahn HS, Baek SM, Choi YJ, et al. 2017 thyroid radiofrequency ablation guideline: Korean Society of Thyroid Radiology. Korean J Radiol 2018;19:632-55.

9. Levit E, Bruners P, Günther RW, Mahnken AH. Bile aspiration and hydrodissection to prevent complications in hepatic RFA close to the gallbladder. Acta Radiol 2012;53:1045-8.

10. Conroy PH, O'Rourke J. Tumescent anaesthesia.
Surgeon 2013;11:210-21.

11. Park HS, Baek JH, Park AW, Chung SR, Choi YJ, Lee $\mathrm{JH}$. Thyroid radiofrequency ablation: updates on innovative devices and techniques. Korean $J$ Radiol 2017;18:615-23.

12. Lim HK, Baek JH, Lee JH, Kim WB, Kim TY, Shong YK, et al. Efficacy and safety of radiofrequency ablation for treating locoregional recurrence from papillary thyroid cancer. Eur Radiol 2015;25:163-70.

13. Laeseke PF, Sampson LA, Brace CL, Winter TC 3rd, Fine JP, Lee FT Jr. Unintended thermal injuries from radiofrequency ablation: protection with 5\% dextrose in water. AJR Am J Roentgenol 2006;186(5 Suppl): S249-54.

14. Raman SS, Lu DS, Vodopich DJ, Sayre J, Lassman C. Minimizing diaphragmatic injury during radio-frequency ablation: efficacy of subphrenic peritoneal saline injection in a porcine model. Radiology 2002; 222:819-23.

15. Liu CH, Yu CY, Chang WC, Dai MS, Hsiao CW, Chou YC, et al. Computed tomographic-guided percutaneous radiofrequency ablation with hydrodissection of hepatic malignancies in the subcapsular location: evaluation of safety and technical efficacy. J Chin Med Assoc 2016;79:93-100.

16. Hisham AN, Lukman MR. Recurrent laryngeal nerve in thyroid surgery: a critical appraisal. ANZ J Surg 2002;72:887-9.

17. Shindo ML, Wu JC, Park EE. Surgical anatomy of the recurrent laryngeal nerve revisited. Otolaryngol Head Neck Surg 2005;133:514-9.

18. Bliss RD, Gauger PG, Delbridge LW. Surgeon's approach to the thyroid gland: surgical anatomy and the importance of technique. World J Surg 2000;24:891-7.

19. Klein JA, Jeske DR. Estimated maximal safe dosages of tumescent lidocaine. Anesth Analg 2016;122:1350-9. 